Journal of Mathematics and Statistics 6 (3): 221-225, 2010

ISSN 1549-3644

(C) 2010 Science Publications

\title{
The Magnetic Field Induced by a Lightning Strike's Indirect Effect Double Exponential Current Waveform
}

\author{
${ }^{1}$ Scott L. Meredith, ${ }^{1}$ Susan K. Earles, ${ }^{1}$ Ivica N. Kostanic and ${ }^{2}$ Niescja E. Turner \\ ${ }^{1}$ Department of Electrical and Computer Engineering, \\ ${ }^{2}$ Department of Physics and Space Sciences, \\ Florida Institute of Technology, Melbourne, Florida, USA
}

\begin{abstract}
Problem statement: Develop a new formula which describes the magnetic field induced by a lightning strike's indirect effect double exponential current waveform. Approach: A novel approach for developing a closed-form solution for the magnetic field from the indirect effect double exponential current waveform will be presented. In the literature, models typically employ the pulse waveform to derive the corresponding electromagnetic fields. However, given the Department of Defense (DoD) has incorporated the double exponential current waveform as part of their "Electromagnetic Environmental Effects Requirements For Systems", we felt it important to develop a solution for the magnetic field which utilized this waveform. In order to facilitate the integration required for deriving the field, Taylor series expansion was used for all variable dependent exponential terms. In many publications, the dipole and monopole techniques have been used when solving for the magnetic field. However, for this study the dipole technique was deemed the preferred method for evaluating the field. A derivation of the magnetic field will be presented along with a graphical illustration of the field's distribution over time. Results: The equation presented utilized Taylor series to augment the integration required to solve for the magnetic field. Conclusion: A new method for deriving the magnetic field induced by a lightning strike's indirect effect double exponential has been presented. By approximating the variable dependent exponential terms, we were able to minimize the complexity of the mathematics required to solve for the magnetic field in closed-form.
\end{abstract}

Key words: Magnetic field, double exponential waveform, lightning and dipole technique

\section{INTRODUCTION}

Bewley (1929) proposed using the double exponential to describe the wave shape a lightning current waveform may have. What he found were measurements obtained in both the field and lab closely matched wave shapes obtained by analysis. This discovery gave rise to an empirical equation which closely resembled lightning's characteristic shape.

Currently, the Department of Defense (DoD) utilizes the double exponential current waveform as part of their Electromagnetic Environmental Effects document MIL-STD-464 to describe the lightning's indirect effect waveform. The parameters $\left(\mathrm{I}_{0}, \alpha\right.$ and $\beta$ ) which make up the waveform are contingent upon the type and severity of the lightning strike. The double exponential current waveform $\mathrm{i}(\mathrm{t})$ described in (MILSTD-464, 1997) is shown by:

$$
\mathrm{i}(\mathrm{t})=\mathrm{I}_{0}\left(\mathrm{e}^{-\alpha \mathrm{t}}-\mathrm{e}^{-\beta \mathrm{t}}\right)
$$

For the purposes of this study we will be utilizing the severe stroke current waveform parameters given in (MIL-STD-464, 1997). The values for $\mathrm{I}_{0}, \alpha$ and $\beta$ are $218,810 \mathrm{~A}, 11,354 \mathrm{~s}-1$ and 647,265 s-1 respectively.

Given this waveform's usage in literature, a validation of the parameters used was warranted. Jia and Xiaoqing (2006) used numerical trial and error to solve the double exponential parameters $\alpha$ and $\beta$. Upon substituting these values into the equation they found that the resulting waveform closely models the one which utilized the fixed parameters. Karwowski and Zeddam (1995) developed a method that utilized the mixed-potential Electric Field Integral Equation (EFIE) in the frequency domain to solve for the electric field numerically using method of moments. However, this study will introduce a closed-form solution for the magnetic field which is induced by the double exponential current waveform.

Corresponding Author: Scott L. Meredith, Department of Electrical and Computer Engineering, Florida Institute of Technology, Melbourne, Florida, USA 


\section{MATERIALS AND METHODS}

Retarded time: In differential form Maxwell's equations for a homogeneous, time variant and linear medium can be written (Jackson, 1999), where D is the electric displacement, $\mathrm{E}$ is the electric field, $\mathrm{B}$ is the magnetic field, $\mathrm{H}$ is the magnetic field strength, $\mathrm{J}$ is the current density, $\rho$ is the charge distribution per unit volume, $\mu_{0}$ the magnetic permeability and $\varepsilon_{0}$ is the electric permittivity:

$\nabla \cdot E=\rho / \varepsilon_{0}$

$\nabla \times \mathrm{E}=-\mu_{0} \frac{\partial \mathrm{H}}{\partial \mathrm{t}}$

$\nabla \times \mathrm{H}=\mathrm{J}+\varepsilon_{0} \frac{\partial \mathrm{E}}{\partial \mathrm{t}}$

$\nabla \cdot \mu_{0} \mathrm{H}=0$

Given Eq. 5, one can solve for the magnetic field in terms of the vector potential alone. Upon utilizing some substitutions and vector identities one would obtain:

$\mu_{0} \mathrm{H}=\nabla \times \mathrm{A}$

where the potential A in terms of z' equals:

$\mathrm{A}_{\mathrm{z}}\left(\mathrm{r}_{\mathrm{s}}, \mathrm{t}\right)=\frac{\mu_{0}}{4 \pi} \int_{\mathrm{z}^{\prime}} \frac{\mathrm{I}\left(\mathrm{r}_{\mathrm{s}}, \mathrm{t}\right) \mathrm{dz}}{\mathrm{R}}$

Upon applying the Curl operator in cylindrical coordinates for (6), we can now re-write the magnetic field such that:

$\mathrm{H}=\frac{1}{\mu_{0}}\left[\frac{1}{\mathrm{r}} \frac{\partial \mathrm{A}_{\mathrm{z}}}{\partial \varphi} \mathrm{i}_{\mathrm{r}}-\frac{\partial \mathrm{A}_{\mathrm{z}}}{\partial \mathrm{r}} \mathrm{i}_{\varphi}\right]$

Given the radial symmetry of the problem, it is apparent that $\mathrm{A}_{\mathrm{z}}$ cannot depend on the coordinate $\varphi$ thus (8) simplifies to:

$\mathrm{H}=-\frac{1}{\mu_{0}} \frac{\partial \mathrm{A}_{\mathrm{z}}}{\partial \mathrm{r}} \mathrm{i}_{\varphi}$

As pointed out by Nevels and Shin (2001), Lorenz proposed that the standard Neumann potentials in terms of instantaneous charge, current density and position be modified to include the propagation of time from the source.
This Retarded time, $\mathrm{t}^{\prime}$ is used as a means of describing the time delay that exists between a photon being emitted and when it is perceived by an observer at a distance $\mathrm{R}$ some R/c time later. The Retarded time or time delay is defined as:

$t^{\prime}=\mathrm{t}-\mathrm{R} / \mathrm{c}$

Where:

$\mathrm{t}^{\prime} \quad=$ The actually time of photon emission

$\mathrm{t}=$ The time it's perceived by an observer

$\mathrm{R} / \mathrm{c}=$ The time it took to travel

The inclusion of the retarded time is used to account for the delay that exists between the lightning channel and observer. By differentiating both sides of (7) and inserting (10) to account for the Retarded time, one could now describe the potential A with:

$\mathrm{dA}_{\mathrm{z}}\left(\mathrm{r}_{\mathrm{s}}, \mathrm{t}\right)=\frac{\mu}{4 \pi} \frac{\mathrm{I}\left(\mathrm{r}_{\mathrm{s}}, \mathrm{t}-\mathrm{R} / \mathrm{c}\right) \mathrm{dz}}{\mathrm{R}}$

Note: By canceling out the areas we can write the current density per unit area $J$ in terms of $I$ and $d V^{\prime}$ in terms of $\mathrm{dz}$.

Upon substituting (11) into (9), one can develop a general expression for the magnetic field at any point in $(\mathrm{r}, \mathrm{z})$ space such that:

$\mathrm{dH}_{\Phi}=\frac{1}{4 \pi}\left[\frac{\mathrm{r}}{\mathrm{cR}^{2}} \frac{\partial \mathrm{i}(\mathrm{t}-\mathrm{R} / \mathrm{c})}{\partial \mathrm{t}}+\frac{\mathrm{r}}{\mathrm{R}^{3}} \mathrm{i}(\mathrm{t}-\mathrm{R} / \mathrm{c})\right] \mathrm{dz^{ \prime }}$

Image theory: Since the conductivity of the earth's surface increases with frequency, it is logical to assume that above a certain frequency the conductivity $\sigma$, approaches infinity. Therefore, if we treat the earth as a perfect conductor, we are able to use image theory in our analysis. These images, or virtual sources, account for reflections which can then be added to the real source constituents to form a general equation. An illustration of this phenomenon is depicted in Fig. 1 which captures the lightning channel and its corresponding image. In order to account for the current waveform that moves upward at a velocity $v$, we now must include the term $\left|z^{\prime}\right| / v$ (Rubinstein and Uman, 1989). With this additional term we can now describe the current waveform (1) with the following:

$$
i\left(t-z^{\prime} / v\right)=I_{0}\left(e^{-\alpha\left(t-z^{\prime} / v\right)}-e^{-\beta\left(t-z^{\prime} / v\right)}\right) \cdot u\left(t-z^{\prime} / v\right)
$$

where, $\left|z^{\prime}\right|$ is written to include all points along the $\mathrm{z}$ axis both real and reflected while the Heaviside function, $\mathrm{u}(\mathrm{t})$ is required to bound the current waveform. 


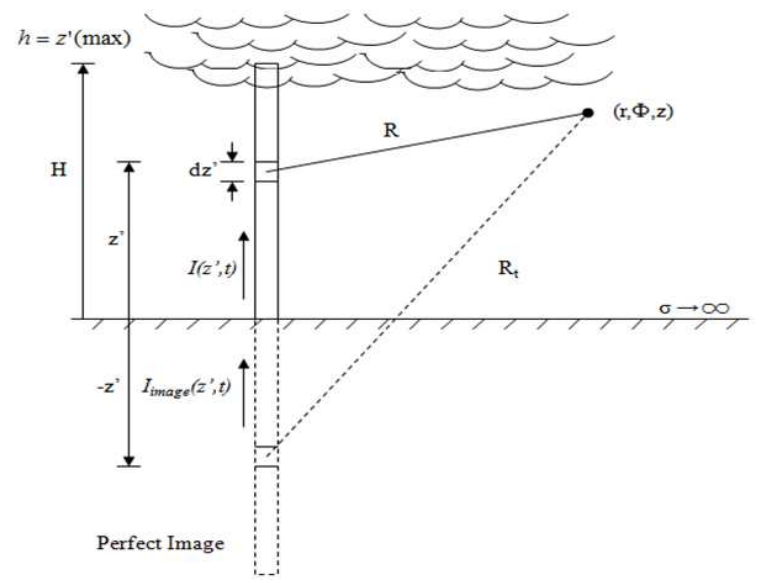

Fig. 1: Application of image theory used for lightning channel

As Rubinstein and Uman (1989) pointed out, by assuming no current flows above height $\mathrm{h}$ in the lightning channel, allows one to set the Heaviside function equal to zero. The known absence of current permits one to conclude that time at this point must equal zero as well. Given the propagating current waveform shifts in time along both the vertical and horizontal axis, allows one to include both of these conditions when defining the time at the top of the channel. With this in mind, one should now encompass the horizontal or "Retarded time" shift, along with the waveform's vertical movement $\left|\mathrm{z}^{\prime}\right| / \mathrm{v}$, to develop a timebased expression at the top of the channel. In doing so, we can expand Eq. 13 to include the Retarded time such that:

$$
\begin{aligned}
\mathrm{i}\left(\mathrm{z}^{\prime}, \mathrm{t}-\mathrm{R} / \mathrm{c}\right)= & \mathrm{I}_{0}\left(\mathrm{e}^{-\alpha\left(\mathrm{t}-\mathrm{R} / \mathrm{c}-\mathrm{z}^{\prime} / \mathrm{v}\right)}-\mathrm{e}^{-\beta\left(t-R / c-z^{\prime} / \mathrm{v}\right)}\right) \\
& \cdot u\left(t-R / c-z^{\prime} / \mathrm{v}\right)
\end{aligned}
$$

Given:

$\mathrm{t}-\mathrm{R} / \mathrm{c}-\left|\mathrm{z}^{\prime}\right| / \mathrm{v}=0$

and

$\mathrm{R}=\sqrt{\left(\mathrm{z}-\mathrm{z}^{\prime}\right)^{2}+\mathrm{r}^{2}}$

one can derive an equation in terms of some height $h$. Plugging (16) into (15), setting z' equal to an arbitrary height $\mathrm{h}$ and solving for $\mathrm{t}$ one would obtain:

$\mathrm{t}=\frac{\sqrt{(\mathrm{z}-\mathrm{h})^{2}+\mathrm{r}^{2}}}{\mathrm{c}}+\frac{\mathrm{h}}{\mathrm{v}}$
After some diligent algebraic manipulations, the height can now be expressed by:

$$
\mathrm{h}=\beta \frac{(\mathrm{ct}-\beta \mathrm{z}) \pm \sqrt{(\mathrm{z}-\beta \mathrm{ct})^{2}+\left(1-\beta^{2}\right) \mathrm{r}^{2}}}{1-\beta^{2}}
$$

where the quantity $\beta=\mathrm{v} / \mathrm{c}$ is the ratio of the current propagation speed along the lightning channel and the speed of light. Given the formula is in quadratic form, two possible solutions exist for $h$. One solution is Causal while the other is non-Causal. Causal signals are zero for all negative values of $t$ while non-Causal signals are zero for all positive values of $t$. In this case, only the Causal form of $\mathrm{h}$ will be considered as one of the participating parameters for evaluating the magnetic field.

Approximating the double exponential: Given the complexity of the integration required to solve for the magnetic field, we must separate the exponential terms into their constant and variable constituents. Once the two have been separated, Taylor series expansion can be used to approximate the exponential terms which contain the variable $z$ '. The expansion for the exponential terms can be approximated by:

$\mathrm{e}^{\mathrm{x}} \approx 1+\mathrm{x}+\frac{\mathrm{x}^{2}}{2 !}+\frac{\mathrm{x}^{3}}{3 !}+\ldots$

In principle, there are two double exponential waveforms that must be approximated. These include: the original waveform and differentiated waveform. Each of which will have been shifted in time by R/c + z'/v. From (14), one can separate the exponentials in terms of their constants and variables constituents. Doing so allows one to utilize Taylor series to account for the variable terms while constants can be set aside and incorporated at a later time. Therefore from (14), we can distribute $\alpha$ and $\beta$ then regroup the exponential terms shown by (20-21):

$$
\begin{aligned}
i\left(z^{\prime}, t-R / c\right)= & I_{0}\left[e^{-\alpha t} \cdot e^{\alpha R / c} \cdot e^{\alpha z^{\prime} / v}-e^{-\beta t} \cdot e^{\beta R / c} \cdot e^{\beta z^{\prime} / v}\right] \\
& \cdot u\left(t-R / c-z^{\prime} / v\right) \\
i\left(z^{\prime}, t-R / c\right)= & I_{0}\left[e^{-\alpha t} \cdot e^{\alpha\left(R / c+z^{\prime} / v\right)}-e^{-\beta t} \cdot e^{\beta\left(R / c+z^{\prime} / v\right)}\right] \\
& \cdot u\left(t-R / c-z^{\prime} / v\right)
\end{aligned}
$$

Given (21), we can make the following assignments:

$$
\mathrm{x}_{\alpha}=\alpha\left(\frac{\mathrm{R}}{\mathrm{c}}+\frac{\mathrm{z}^{\prime}}{\mathrm{v}}\right)
$$




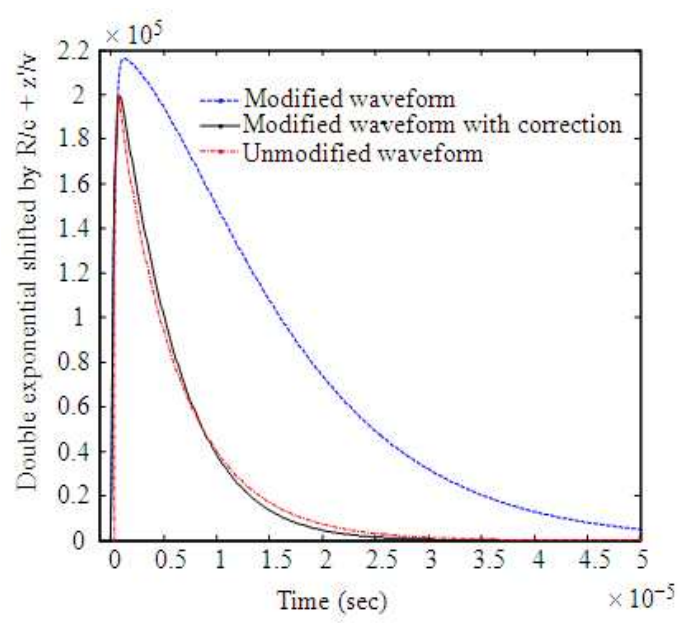

Fig. 2: Modified and unmodified double exponential current waveforms shifted by $\mathrm{t}=\mathrm{R} / \mathrm{c}+\mathrm{z}^{\prime} / \mathrm{v}$

and:

$\mathrm{x}_{\beta}=\beta\left(\frac{\mathrm{R}}{\mathrm{c}}+\frac{\mathrm{z}^{\prime}}{\mathrm{v}}\right)$

Therefore, one can leverage the first two terms from $(19)$ where $(22,23)$ equal the variable $\mathrm{x}$ as given by:

$$
\begin{aligned}
i\left(z^{\prime}, t-R / c\right)=I_{0} & {\left[e^{-\alpha t} \cdot\left(1+\alpha\left(\frac{R}{c}+\frac{z^{\prime}}{v}\right)\right)\right.} \\
& \left.-e^{-\beta t} \cdot\left(1+\beta\left(\frac{R}{c}+\frac{z^{\prime}}{v}\right)\right)\right] \\
& \cdot u\left(t-R / c-z^{\prime} / v\right)
\end{aligned}
$$

Upon regrouping the "like" terms, (24) can now be written as:

$$
\begin{aligned}
i\left(z^{\prime}, t-R / c\right)= & I_{0}\left[e^{-\alpha t}-e^{-\beta t}+\left(\alpha e^{-\alpha t}-\beta e^{-\beta t}\right)\right. \\
& \left.\cdot\left(\frac{R}{c}+\frac{z^{\prime}}{v}\right) \cdot u\left(t-R / c-z^{\prime} / v\right)\right]
\end{aligned}
$$

with differentiated form of (25) being:

$$
\begin{aligned}
i\left(z^{\prime}, t-R / c\right)= & I_{0}\left[\beta e^{-\beta t}-\alpha e^{-\alpha t}+\left(\beta^{2} e^{-\beta t}-\alpha^{2} e^{-\alpha t}\right)\right. \\
& \left.\cdot\left(\frac{R}{c}+\frac{z^{\prime}}{v}\right) \cdot u\left(t-R / c-z^{\prime} / v\right)\right]
\end{aligned}
$$

As the Fig. 2 shows, the modified waveform which utilized Taylor series expansion tends to yield a slightly larger magnitude and takes longer to decay. However, when this approximated waveform is modified with the seminal constituent $1.06 * \exp (-1.25 \alpha \mathrm{t})$, the resulting solution reveals a waveform that closely models the original double exponential shifted by $R / c+z / v$. By realizing the acquiescent similarities between the original and approximated waveforms allows one to leverage the latter to derive the magnetic field in closed-form with a high level of confidence.

\section{RESULTS}

Calculating the fields: The methodology used in deriving the magnetic field will forgo using the standard square pulse presented in literature (Rubinstein and Uman, 1989) and replace it with the double exponential waveform. Let's first start off by rewriting (12) such that:

$$
\mathrm{dH}_{\Phi}=\frac{1}{4 \pi}\left[\frac{\mathrm{r}}{\mathrm{cR}^{2}} \frac{\partial \mathrm{i}(\mathrm{t}-\mathrm{R} / \mathrm{c})}{\partial \mathrm{t}}+\frac{\mathrm{r}}{\mathrm{R}^{3}} \mathrm{i}(\mathrm{t}-\mathrm{R} / \mathrm{c})\right] \mathrm{dz^{ \prime }}
$$

Next, substitute $(25,26)$ into the second and first terms of (27) respectively. Upon doing so, one will arrive at:

$$
\begin{aligned}
\mathrm{dH}_{\Phi}=\frac{\mathrm{I}_{0} \mathrm{dz}}{4 \pi}\left[\frac { \mathrm { r } } { \mathrm { cR } \mathrm { R } ^ { 2 } } \left(\beta \mathrm{e}^{-\beta \mathrm{t}}-\alpha \mathrm{e}^{-\alpha \mathrm{t}}+\left(\beta^{2} \mathrm{e}^{-\beta \mathrm{t}}-\alpha^{2} \mathrm{e}^{-\alpha \mathrm{t}}\right)\right.\right. \\
\left.\cdot\left(\frac{\mathrm{R}}{\mathrm{c}}+\frac{\mathrm{z}^{\prime}}{\mathrm{v}}\right)\right) \cdot \mathrm{u}\left(\mathrm{t}-\mathrm{R} / \mathrm{c}-\mathrm{z}^{\prime} / \mathrm{v}\right) \\
+\frac{\mathrm{r}}{\mathrm{cR} \mathrm{R}^{2}}\left(\mathrm{e}^{-\alpha \mathrm{t}}-\mathrm{e}^{-\beta \mathrm{t}}+\left(\alpha \mathrm{e}^{-\alpha \mathrm{t}}-\beta \mathrm{e}^{-\beta \mathrm{t}}\right)\right. \\
\left.\cdot\left(\frac{\mathrm{R}}{\mathrm{c}}+\frac{\mathrm{z}^{\prime}}{\mathrm{v}}\right)\right) \cdot \delta\left(\mathrm{t}-\mathrm{R} / \mathrm{c}-\mathrm{z}^{\prime} / \mathrm{v}\right) \\
+\frac{\mathrm{r}}{\mathrm{R}^{3}}\left(\mathrm{e}^{-\alpha \mathrm{t}}-\mathrm{e}^{-\beta \mathrm{t}}+\left(\alpha \mathrm{e}^{-\alpha \mathrm{t}}-\beta \mathrm{e}^{-\beta \mathrm{t}}\right)\right. \\
\left.\left.\cdot\left(\frac{\mathrm{R}}{\mathrm{c}}+\frac{\mathrm{z}^{\prime}}{\mathrm{v}}\right)\right) \cdot \mathrm{u}\left(\mathrm{t}-\mathrm{R} / \mathrm{c}-\mathrm{z}^{\prime} / \mathrm{v}\right)\right]
\end{aligned}
$$

Note the additional term added to (28) is due in part to the following product rule:

$[\mathrm{f}(\mathrm{t}) \cdot \mathrm{u}(\mathrm{t})]^{\prime}=\mathrm{f}(\mathrm{t})^{\prime} \cdot \mathrm{u}(\mathrm{t})+\mathrm{f}(\mathrm{t}) \cdot \delta(\mathrm{t})$

Although the terms bound by the delta function are shown in (28), they vanish once the current waveforms are evaluated at $\mathrm{t}=\mathrm{R} / \mathrm{c}+\mathrm{z}^{\prime} / \mathrm{v}$. Upon integrating Eq. 28 from $-\mathrm{h}$ to $\mathrm{h}$ along the $\mathrm{z}=0$ plane, we can obtain a 
closed-form solution for the magnetic field to include the correction factor as shown by the following:

$$
\begin{aligned}
& \mathrm{H}_{\Phi}(\mathrm{r}, 0, \mathrm{t})=\frac{\mathrm{I}_{0}\left(1.06 \mathrm{e}^{-1.25 \alpha \mathrm{t}}\right)}{2 \pi}\left[\frac{\mathrm{r}\left(\beta^{2} \mathrm{e}^{-\beta \mathrm{t}}-\alpha^{2} \mathrm{e}^{-\alpha \mathrm{t}}\right)}{\mathrm{c}^{2}}\right. \\
& \cdot \ln \left|\frac{\sqrt{\mathrm{h}^{2}+\mathrm{r}^{2}}}{\mathrm{r}}+\frac{\mathrm{h}}{\mathrm{r}}\right| \\
&-\frac{\mathrm{r}\left(\beta^{2} \mathrm{e}^{-\beta \mathrm{t}}-\alpha^{2} \mathrm{e}^{-\alpha \mathrm{t}}\right)}{\mathrm{cv}} \cdot \ln \left|\frac{\mathrm{r}}{\sqrt{\mathrm{h}^{2}+\mathrm{r}^{2}}}\right| \\
&+\frac{\left(\mathrm{e}^{-\alpha \mathrm{t}}-\mathrm{e}^{-\beta \mathrm{t}}\right)}{\mathrm{r}} \cdot \frac{\mathrm{h}}{\sqrt{\mathrm{h}^{2}+\mathrm{r}^{2}}} \\
&\left.+\frac{\left(\alpha \mathrm{e}^{-\alpha \mathrm{t}}-\beta \mathrm{e}^{-\beta \mathrm{t}}\right)}{\mathrm{v}} \cdot\left(1-\frac{\mathrm{r}}{\sqrt{\mathrm{h}^{2}+\mathrm{r}^{2}}}\right)\right]
\end{aligned}
$$

Figure 3 illustrates how the magnetic field's magnitude is distributed as time varies from $0-1 \times 10^{-5}$ sec.

\section{DISCUSSION}

Equation 30 used a methodology which greatly simplified the integration required to solve for the magnetic field in a closed-form. By utilizing Taylor series to approximate the exponential terms, the solution's accuracy became a function of the number of terms used from (19). Consequently, a correction factor needed to be added in order to help reshape the magnetic field which resulted from the modified current waveform.

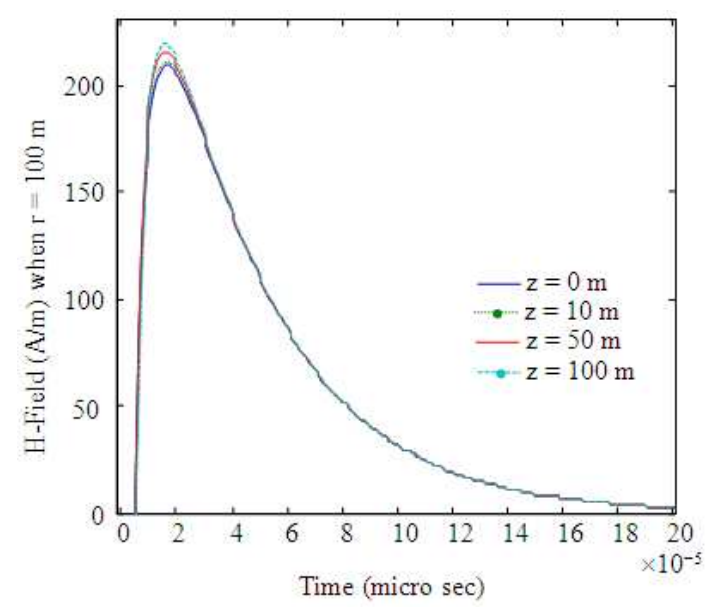

Fig. 3: Magnetic fields from the approximated double exponential current waveforms at various heights $\mathrm{z}$

\section{CONCLUSION}

A closed-form solution for the magnetic field induced by the double exponential current waveform has been presented. The approach proposed here is unique in that it utilized Taylor series to approximate the variable dependent exponential terms within the current waveform. This greatly simplified the mathematics which helped facilitate the integration required to solve for the solution in a closed-form. However, this approximation caused the modified current waveform to grow in both magnitude and decay time. With this in mind, it is logical to presume that the resulting fields would be subject to this same anomaly. Realizing a departure existed between the modified and unmodified waveforms, warranted the use of a correction factor to recapture its original form. Doing so ensured the magnetic fields which followed, would tend to more closely model those from the original unmodified current waveform.

\section{REFERENCES}

Bewley L.V., 1929. Traveling waves due to lightning. AIEE Trans., 49: 1050-1064.

Department of Defense Interface Standards, 1997. Electromagnetic Environmental Effects Requirements for Systems. MIL-STD-464, pp: 1-10.

Jackson, J.D., 1999. Classical Electrodynamics. 3rd Edn., John Wiley and Sons Inc, New Jersey, USA, ISBN: 0-471-30932-X, pp: 24-63.

Jia, W. and Z. Xiaoqing, 2006. Double-Exponential Expression of Lightning Current Waveforms. CEEM Dalian 3A1-09, pp: 320-323.

Karwowski A. and A. Zeddam, 1995. Transient currents on lightning protection systems due to the indirect effect. IEE Proc. Sci. Meas. Technol., 142: 231-222.

Nevels, R. and C. Shin, 2001. Lorenz, Lorentz and the gauge. IEEE Antenn. Propagat. Mag., 43: 70-72.

Rubinstein, M. and M.A. Uman, 1989. Methods for calculating the electromagnetic fields from a known source distribution: Application to lightning. IEEE Trans. Elect. Compat., 31: 183-189. 